\title{
The Genomic Characteristics of RET Fusion Positive Tumors in Chinese Non-small Cell Lung Cancer (NSCLC) Patients
}

Guowu Wu ( $\nabla$ guowuwugd@163.com )

Yan an People's Hospital: Yan'an People's Hospital

Longhua Guo

Meizhou People's Hospital(Huangtang Hospital)

\section{Yinfang Gu}

Meizhou People's Hospital(Huangtang Hospital)

Tanxiao Huang

HaploX Biotechnology Co., Ltd.

Ming Liu

HaploX Biotechnology

Xiaofang Zou

Meizhou People'sHospital(Huangtang Hospital)

Bo Yang

HaploX Biotechnology

Ping Huang

Meizhou People's Hospital(Huangtang Hospital)

Chunling Wen

Meizhou People's Hospital(Huangtang Hospital)

Lilan Yi

Meizhou people's Hospital(Huangtang Hospital)

Wenting Liao

Haplox Biotechnology

Dongdong Zhao

Meizhou people's Hospital(Huangtang Hospital)

Junlin Zhu

Meizhou People's Hospital(Huangtang Hospital)

Xiaoni Zhang

Haplox Biotechnology

Yuanyuan Liu

Haplox Biotechnology

Yan Yin 
Haplox Biotchnology

\section{Shifu Chen}

HaploX Biotchnology Co., Ltd.,

\section{Research}

Keywords: RET, Non-Small Cell Lung Cancer, Next Generation Sequencing

Posted Date: July 28th, 2021

DOl: https://doi.org/10.21203/rs.3.rs-655553/v1

License: (1) This work is licensed under a Creative Commons Attribution 4.0 International License. Read Full License 


\section{Abstract}

Background: Approximately $1-2 \%$ of non-small cell lung cancer (NSCLC) patients harbor RET (rearranged during transfection) fusions. The oncogenic RET fusions could lead to constitutive kinase activation and oncogenesis.

Methods: 1746 Chinese NSCLC patients were enrolled from in this study. Tumor tissues were collected during surgery, and were formalin fixed, paraffin-embedded (FFPE) and archived. Peripheral blood (PB) samples were also collected from each patient as control. In addition, we selected 17 of them for cfDNA NGS testing and 14 tumor samples for immunohistochemistry testing using PD-L1 rabbit monoclonal antibody, clones 28-8 (Abcam, Cambridge, UK).

Results: Of the 1746 NSCLC cases, RET rearrangements were identified in 25 cases (1.43\%) with locally advanced or metastatic NSCLC, of which $20(80 \%)$ were female.We found that 14 out of 25 patients had an KIF5B-RET fusion, with KIF5B exon15-RET exon12, KIF5B exon23-RET exon12, and KIF5B exon24-RET exon 11 detected in 14, 3 , and 1 patients respectively. We also identified one novel RET fusion partner PLCE1 and 4 intergenic-breakpoint fusions.

Conclusion: In this study, using the hybrid capture based NGS (the next generation sequencing) techniques, we revealed the genomic profiling for the patients with RET fusion-positive NSCLC. To the best of our knowledge, this is the first study that exhibited the detailed breakpoints of Chinese NSCLC patients with RET rearrangement, and we found a novel new partner PLCE1. This result provides genomic information for personalized clinical management for patients with RET fusion in the era of precision medicine.

\section{Background}

It was not until 2012 that the RET fusions were identified as oncogenic drivers in NSCLC [1]. Approximately 1-2\% of NSCLC patients harbor RET fusions [2], and they tend to be young, non-smokers, and adenocarcinomas[3]. The RET proto-oncogene encodes a transmembrane receptor tyrosine kinase belonging to members of the glial cell line-derived neurotrophic factor (GDNF) family. The RET signaling is modulated by ligands and could activate multiple downstream pathways, such as RAS/MAPK/ERK, PI3K/AKT and JAK/STAT, which are essential for cellular differentiation and proliferation. However, the oncogenic RET fusions could lead to constitutive kinase activation and oncogenesis [4-6]. RET fusions are caused by chromosomal rearrangement, which fuses the $3^{\prime}$ coding regions for the RET kinase domain on chromosome 10 with a $5^{\prime}$ upstream partner gene containing coiled-coil or LISI homology domains [79]. The most common partner genes are KIF5B, CCDC6 and NCOA4, which originate from intrachromosomal rearrangements, but there are also some relatively infrequent interchromosomal partner genes, such as TRIM33, TRIM24, MY05C, EPHA5, CLIP1, and so on [10,11].

The advent of precision medicine has revolutionized the therapeutic landscape of NSCLC, and targeted therapies have been investigated in many clinical studies against patients with RET fusion-positive 
NSCLC , which includes multi-kinase inhibitors (MKIs) and selective RET inhibitors [12]. MKIs target not only RET, but also other kinases, such as VEGFR2, KIT, BRAF, etc. This probably leads to decreased effectiveness against RET and off-target side effects. Several MKIs that were approved by FDA for cancer therapy on other purposes (such as sunitinib, sorafenib, vandetanib, cabozantinib, regorafenib, lenvatinib, and alectinib) all showed modest clinical activity and had side effects for patients with RET fusionpositive NSCLC[13-18]. Besides, MKI resistance is almost inevitable. The common resistant mechanisms are secondary MET gene mutation and downstream signaling pathway activation $[19,20]$. These limitations in MKIs prompt the development of selective RET inhibitors. On May 8, 2020, the FDA granted accelerating approval to selpercatinib for adult patients with metastatic RET fusion-positive NSCLC. This was the first targeted therapy approved for RET fusion-positive NSCLC [21]. The other selective RET inhibitor, pralsetinib, was also granted as Breakthrough Therapy designation by the FDA for advanced NSCLC with RET fusions after progression on platinum chemotherapy. Both of them showed higher response rates and tolerability, but drug resistance were also inevitable for them $[22,23]$.

The development of hybrid capture-based NGS techniques brings great convenience in revealing the genomic profiling for cancer patients. It's becoming more and more available in clinical cancer treatment. It not only could be used to identify the RET fusion events, but also allows us to investigate the cooccurring genomic alterations in the same assay, which may be related to prognosis or therapeutic response. In this study, we sequenced 1746 NSCLC patients by hybrid capture-based NGS techniques, and eventually identified 25 RET fusion-positive cases.

\section{Materials And Methods}

\section{Patients and samples}

1746 Chinese NSCLC patients were enrolled from in this study. Tumor tissues were collected during surgery, and were formalin fixed, paraffin-embedded (FFPE) and archived. Peripheral blood (PB) samples were also collected from each patient as control. In addition, we selected 17 of them for cfDNA NGS testing and 14 tumor samples for immunohistochemistry testing using PD-L1 rabbit monoclonal antibody, clones 28-8 (Abcam, Cambridge, UK).

\section{FFPE DNA extraction}

DNA samples were extracted from Formalin-fixed paraffin-embedded (FFPE) samples with QIAamp DNA FFPE tissue kit (Qiagen). Extraction of PBL DNA was conducted using the RelaxGene Blood DNA system (Tiangen Biotech Co., Ltd., Beijing, China) according to the manufacturer's protocol. All the DNA samples were quantified using both the Qubit 2.0 fluorometer and the Qubit dsDNA HS Assay kit (Thermo Fisher Scientific, Inc., Waltham, MA, USA) according to the manufacturer's protocol.

\section{Plasma isolation and cfDNA extraction.}


Blood samples from patients were collected in tubes containing EDTA and centrifuged at $1600 \mathrm{~g}$ for 10 min at $4{ }^{\circ} \mathrm{C}$ within 2 hours of collection. The peripheral blood lymphocyte (PBL) debris was stored at $-20^{\circ} \mathrm{C}$ until further use. The supernatants were further centrifuged at $10,000 \mathrm{~g}$ for 10 min at $4{ }^{\circ} \mathrm{C}$, and plasma was harvested and stored at $-80^{\circ} \mathrm{C}$ until further use. DNA from PBLs was extracted using RelaxGene Blood DNA System(TianGen Biotech Co., Ltd., Beijing, China), and cell free DNA(cfDNA) was extracted from at least $2 \mathrm{~mL}$ plasma using QIAamp Circulating Nucleic Acid kit (QIAGEN) following the manufacturers' instructions, respectively. Extracted DNA was then quantified by Qubit 2.0 (Thermo Fisher Scientific, Inc., Waltham, MA, USA), according to manufacturer's instructions.

\section{FFPE and genomic DNA Library construction and sequencing}

100ng of FFPE DNA and genomic DNA from PBLs for each patient was sheared by the dsDNA Fragmentase (New England BioLabs, Inc., Ipswich, MA, USA), and then size selection (150-250 bp) was performed using Ampure XP beads (Beckman Coulter, Inc., Brea, CA, USA). Library construction was performed using the KAPA Library Preparation kit (Kapa Biosystems, Inc., Wilmington, MA, USA) according to the manufacturer's protocol. The concentration of the library were assessed using the $\mathrm{e}$ Qubit dsDNA HS Assay kit, and fragment length was determined on a 4200 Bioanalyzer (Agilent Technologies, Inc., Santa Clara, CA, USA). Targeted capture was performed using a custom set of biotinylated DNA probes(HapOncoCDx panel) which contain 464 cancer-related genes encompassing $1.31 \mathrm{Mb}$ (Roche NimbleGen). The hybridization of the amplified sample libraries and the SeqCap EZ Library was used according to the manufacturer's protocol for $16-20 \mathrm{~h}$ at $47^{\circ} \mathrm{C}$. After hybrid selection, the captured DNA fragments were amplified with 12 to 14 cycles of PCR using $1 \times$ KAPA HiFi Hot Start Ready Mix and Post-LM-PCR Oligos in two separate $50 \mu \mathrm{L}$ reactions. The reactions were then pooled and purified by Agencourt AMPure XP beads. DNA sequencing was then performed on the Illumina Novaseq 6000 system at an average depth of $2000 \mathrm{X}$

\section{CfDNA Library construction and sequencing}

CfDNA were used for library construction using the KAPA Library Preparation kit (Kapa Biosystems, Inc., Wilmington, MA, USA) according to the manufacturer's protocol. Agencourt AMPure XP beads (Beckman Coulter, Inc., Brea, CA, USA) were used for all the cleanup steps. The purity and concentration of the DNA fragments were assessed using the Qubit 2.0 fluorometer and the Qubit dsDNA HS Assay kit. End repair and 3 '-end A-tailing were performed following DNA fragmentation. Ligation was performed for $15 \mathrm{~min}$ at $20^{\circ} \mathrm{C}$. Single-step size selection was performed by adding $50 \mu \mathrm{L}(1 \times)$ of PEG/NaCl SPRI Solution buffer to enrich for ligated DNA fragments. The ligated fragments were then amplified using $1 \times$ KAPA HiFi Hot Start Ready Mix and Pre-LM-PCR Oligos in 50 $\mu \mathrm{L}$ reactions, and 7-12 PCR cycles were performed depending on quantity of input DNA . Library's purity and concentration was assessed by Qubit 2.0 Fluorometer and the Qubit dsDNA HS Assay kit. Fragment length was determined on a 4200 Bioanalyzer using the DNA 1000 Kit (Agilent).

Targeted capture was conducted using customed HapOncoCDx panel. The hybridization of the amplified sample libraries and the SeqCap EZ Library was used according to the manufacturer's protocol for 16-20h 
at $47^{\circ} \mathrm{C}$. After hybrid selection, the captured DNA fragments were amplified with 12 to 14 cycles of PCR using 1× KAPA HiFi Hot Start Ready Mix and Post-LM-PCR Oligos in two separate $50 \mu \mathrm{L}$ reactions. The reactions were then pooled and purified by Agencourt AMPure XP beads.

Multiplexed libraries were denatured by Tris- $\mathrm{HCl}$ and diluted by $0.2 \mathrm{~N} \mathrm{NaOH}$ according to the manufacturer's protocol (Illumina). Then the libraries were sequenced using 150-bp paired-end runs on an Illumina NovaSeq 6000 system (Illumina).

\section{Data analysis and variant calling}

Raw sequences were pre-processed by fastp version 0.18 .0 (https://github.com/OpenGene/fastp) [24], and clean reads were aligned to the hg19 genome (GRch37) using Burrows-Wheeler Aligner maximal exact matches algorithm [25]. The Gencore version 0.12.0(https://github.com/OpenGene/gencore) [26] was used for removing duplicate reads. Pileup files with mapping quality $\geq 60$ were generated using Samtools version 0.1.19(http://www.htslib.org/) [27]. Somatic variants were called using VarScan2 version 2.3.8 (http://varscan.sourceforge.net/) [28] [the minimum read depth 20; the variant allele frequency (VAF) threshold $\geq 0.01$; somatic-P-value $\leq 0.01$; strand-filter $=1$; others, default parameters]. CNV kit with version 0.9.3(https://github.com/etal/cnvkit) [29] were applied for copy number variation detection, and GeneFuse version v0.6.1 (https://github.com/OpenGene/GeneFuse) [30] for structural variation detection. Microsatellite instability (MSI) status were determined by VisualMSI(https://github.com/OpenGene/VisualMSI)[31]. Maftools were used for visualizing somatic variant analysis [32]. Data which meet the following criteria were chosen for subsequent analysis: the ratio of remaining data filtered by fastq in raw data is $\geq 85 \%$; the proportion of Q30 bases is $\geq 85 \%$; the ratio of reads on the reference genome is $\geq 85 \%$; target region coverage $\geq 98 \%$; average sequencing depth in tissues is $\geq 500 x$; average sequencing depth in blood cfDNA is $\geq 1000 \times$. The called somatic variants need to meet the following criteria: the read depth at a position is $\geq 100 x$; the variant allele frequency (VAF) is $\geq 2 \%$ for tissue DNA and $\geq 0.2 \%$ for cfDNA from blood; somatic-P $\leq 0.01$; strand filter $=1$. Allele frequencies were calculated for Q30 bases. For cfDNA, somatic variant calls (SNV or indel) present at least on 5 unique reads, at least 1 on each strand, and less than $0.5 \%$ mutant allelic frequency in the paired normal sample (PBLs) were retained. A manual visual inspection step was used to further remove artifactual changes by GenomeBrowse [33].

\section{Results}

\section{Sample collection and patient characteristics}

Of the 1746 NSCLC cases, RET rearrangements were identified in 25 cases (1.43\%) with locally advanced or metastatic NSCLC, of which $20(80 \%)$ were female. The 25 patients have mean age of 53.5 ranging from 27 to 78. NGS testing was performed on their 25 pairs of FFPE tumor tissue and PBL samples. In addition, we performed cfDNA NGS testing on 17 of them to check their RET gene status in cfDNA sample. All the samples passed the histology quality control (HQC) and yielded sufficient amounts of DNA for NGS. 


\section{Identification of RET rearrangements using targeted sequencing}

In order to identify RET rearrangement of the DNA from patients' FFPE samples, we designed probes to cover the intron $6,7,8,9,10,11$ of RET as well as introns of some well-known RET fusion partners. We identified RET rearrangements and corresponding breakpoints in the sequencing data of these patients. The statistical summary of the rearrangement events are presented in Table 1 and in Figure 1. The breakpoint distribution in RET are shown in Figure 2 . We found that 14 out of 25 patients had an KIF5BRET fusion, with KIF5B exon15-RET exon12, KIF5B exon23-RET exon12, and KIF5B exon24-RET exon11 detected in 14, 3 , and 1 patients respectively. We also identified one novel RET fusion partner PLCE1 and 4 intergenic-breakpoint fusions.

Table 1. Fusion patterns of RET

\begin{tabular}{|lll|}
\hline Fusion Type & Counts & percent(\%) \\
\hline KIF5B-exon15-RET-exon12 & 14 & 56 \\
\hline CCDC6-exon1-RET-exon12 & 3 & 12 \\
\hline KIF5B-exon23-RET-exon12 & 1 & 4 \\
\hline KIF5B-exon24-RET-exon11 & 1 & 4 \\
\hline PLCE1-exon20-RET-exon11 & 1 & 4 \\
\hline RET-exon11-CCDC6-exon3 & 1 & 4 \\
\hline Other & 4 & 16 \\
\hline
\end{tabular}

\section{Mutational profiles of RET fusion-positive NSCLC patients}

Genomic alterations were found in $24(n=24 / 25,96 \%)$ samples with a total of 113 alterations identified including variants of nonsynonymous mutations and splicing mutations. The top 20 alterations are chosen to present in Figure 3A. The mutation landscapes of RET fusion-positive NSCLC patients were highly heterogeneous. The median TMB was 2.4 mut/ $\mathrm{Mb}$ with a range between 0 to 8.4 mut/Mb, which is similar to the TMB of the TCGA NSCLC cohort[35].

We constructed a heatmap to demonstrate the somatic mutations occurring in the tumor tissues of the patients (Figure 3A). TP53 was most commonly altered $(n=10,42 \%)$, followed by SETD2 $(n=4,17 \%)$, CSMD3 $(n=3,12 \%)$, and PTEN $(n=3,12 \%)$. Other genomic alterations with low frequencies are ATM $(n=$ $2,6 \%), \operatorname{CACNA} 1 \mathrm{C}(\mathrm{n}=2,8 \%), \operatorname{CIC}(n=2,8 \%), \operatorname{CTCF}(n=2,8 \%)$, DOT1L $(n=2,8 \%), \operatorname{FANCA}(n=2,8 \%)$, FANCG $(n=2,8 \%), \operatorname{LRP} 1 B(n=2,8 \%)$, MAP2K4 $(n=2,8 \%), \operatorname{NOTCH} 1(n=2,8 \%), \operatorname{PRKCl}(n=2,8 \%)$, PTPRT $(n=2,8 \%)$, RB1 $(n=2,8 \%)$, SMAD4 $(n=2,8 \%)$, and SUZ12 $(n=2,8 \%)$. Alterations in DICER1 were identified in one sample $(n=1,4 \%)$. We further compared our results with the MSK-IMPACT study [32], in which we extracted 30 RET fusion positive cases that yielded 81 mutations. Overall, our results were 
highly consistent with the MSK-IMPACT findings, which showed that TP53 and SETD2 are the most frequently altered genes (Fig. 3B).

We further studied their mutational signatures. We observed that $\mathrm{C}>\mathrm{T}$ transition occurred most frequently, followed by $C>G$ transversions (Fig. 4). This pattern is consistent with COSMIC signature 84 according to website(https://cancer.sanger.ac.uk/signatures/sbs/sbs84/) that had been found in some cancer samples.

Different driver gene mutations revealed inter-tumor heterogeneity. TP53 mutations in exon 4-8 were observed, and we further elaborately portrayed the TP53 mutation sites on the peptide sequence in a lollipop plot. (Fig. 5).

\section{Copy number aberrations of RET fusion-positive NSCLC patients}

Somatic copy number alterations were found in $11(n=11 / 25,44 \%)$ samples. A total of 22 alterations were identified, including gain and loss (Figure 6). CDK4 were most commonly amplified genes $(n=3 / 11$, $27 \%)$. Loss of copy number was most frequently observed in FGFR3 $(n=4 / 11,36 \%)$.

\section{PD-L1 expression and Microsatellite Instability (MSI) status of RET fusion-positive NSCLC patients}

High ( $\geq 50 \%)$, intermediate (1-49\%), and negative (< 1\%) PD-L1 expression was observed in 0/14 (0\%), $8 / 14(57 \%)$, and 6/14(43\%) cases, respectively.

MSI status were evaluated in 20 cases. They are all microsatellite stable (MSS).

\section{Concordance in tumor DNA(tDNA) and plasma DNA(ctDNA) sample pairs of RET fusion-positive NSCLC patients}

A total of 17 tDNA and ctDNA sample pairs were analyzed. 9 patients are found to have the same breakpoint of RET fusions in both tDNA and ctDNA samples, indicating 52.9\% RET fusion could be detected in ctDNA. A total of 111 mutations (snv and indel) were identified, including 90 in tDNA and 64 in plasma ctDNA, and 43 concordant mutations in both tDNA and plasma ctDNA. Seven sample pairs $(7 / 17,41.2 \%)$ had concordant mutations in both tDNA and plasma ctDNA , and the average variant frequency in these plasma ctDNA samples was $8.17 \%$.

\section{Discussion}

In this study, we identified RET rearrangement events in 25 Chinese NSCLC patients by hybrid capture based NGS. Consistent with other reports, the most common RET fusion partner was KIF5B and CCDC6, and the breakpoints in the genome mainly located in the intron 11 of RET, intron 15 of KIF5B, and intron 1 of CCDC6. Amazingly, we discovered a new RET fusion partner PLCE1. Besides, we identified 4 intergenicbreakpoint fusions in 4 cases. A study by Weihua Li reported that intergenic-breakpoint fusions might also generate functional fusion transcripts [36], so additional validation testing such as RNA-seq or IHC 
was required for these patients to guide treatment. At the same time, we checked the concordance of RET fusions between tDNA and cfDNA for the same patient. In most cases, they harbored the same breakpoint, this clearly proved that the fragments of DNA harboring RET fusions were derived from tDNA. Therefore, cfDNA is an excellent alternative material for patients who have difficulty in obtaining tumor tissues. These results also implied that NGS-based assessment for RET fusions had the advantages of detecting unknown RET fusion partners and identifying the same breakpoints as the traditional diagnostic testing, such as FISH and IHC.

At the same time, we characterized the co-occurring genomic alterations of these RET fusion-positive patients. The results were consistent with the TCGA cohort, in terms of the relatively higher frequency of TP53 mutations, lower TMB, and fewer co-mutations compared to RET fusion-negative NSCLC patients. Besides, we identified the copy number alterations in their genome. Apart from the genes with a high frequency of copy number amplification, such as CDK4, we also identified some genes with copy number loss, such as FGFR3. This information is important for guiding optimal clinical treatment.

\section{Conclusion}

In conclusion, using our customized HapOncoCDx panel, we not only successfully detected the RET fusion events in 25 Chinese NSCLC patients, but also explored their genomic mutational landscapes. To the best of our knowledge, this is the first study that exhibited the detailed breakpoints of Chinese NSCLC patients with RET rearrangement, and we found a novel new partner PLCE1. This result provides genomic information for personalized clinical management for patients with RET fusion in the era of precision medicine.

\section{Abbreviations}

Non-small cell lung cancer (NSCLC)

Multi-kinase inhibitors (MKIs)

Formalin-fixed paraffin-embedded (FFPE)

peripheral blood lymphocyte (PBL)

Cell free DNA(cfDNA)

Microsatellite Instability (MSI)

\section{Declarations}

\section{Availability of data and materials}


The datasets for this manuscript are not publicly available due to data privacy. Requests to access the datasets should be directed to the corresponding authors Guowu Wu(guowuwugd@163.com), Longhua Guo(1851258807@qq.com), and Shifu Chen(chen@haplox.com).

\section{Ethics Statement}

The study was approved by the ethics committee of The Meizhou People's Hospital (Huangtang Hospital) and complied with Good Clinical Practices, the principles of the Declaration of Helsinki and all applicable regulatory requirements. All patients provided written informed consent prior to any study-specific procedures.

\section{Authors' Contributions}

Guowu Wu, and Longhua Guo contributed equally to this study. GW, LG, and SC designed the study. YG, $X Z, P H, C W, L Y, D Z, J Z, X Z, Y L$ carried out the sequencing experiment and collected data. TH, ML, WL, and BY performed the

bioinformatics analysis. GW, LG, and TH wrote this manuscript. YY and SC revised this manuscript. GW, LG and SC supervised the study.

\section{Funding}

This work was supported by the project of Medical Science and Technology Research

Foundation of Guangdong Province (Grant No. B2021118), the project of Shenzhen Science and Technology Innovation Committee Technical Research Project (Grant No. KQJSCX20180330124428928 and JSGG20180508152646606), the project of Development and Reform Commission of Shenzhen Municipality (Grant No. XMHT20190104006 and XMHT20200104013).

\section{Competing interests}

TH, ML, BY, WL, XZ, YL, YY and SC were employed by the company HaploX

Biotechnology Co., Ltd. The remaining authors declare that the research was conducted in the absence of any commercial or financial relationships that could be construed as a potential conflict of interest.

\section{Acknowledgements}

None

\section{References}

1. Ju YS, Lee WC, Shin JY, et al. A transforming KIF5B and RET gene fusion in lung adenocarcinoma revealed from whole-genome and transcriptome sequencing. Genome Res. 2012;22(3):436-45. 
2. Cancer Genome Atlas Research Network. Comprehensive molecular profiling of lung adenocarcinoma. Nature. 2014;511(7511):543-50.

3. Wang R, Hu H, Pan Y, et al. RET fusions define a unique molecular and clinicopathologic subtype of non-small-cell lung cancer. J Clin Oncol. 2012;30:4352-9.

4. Worby CA, Vega QC, Zhao Y, et al. Glial cell line-derived neurotrophic factor signals through the RET receptor and activates mitogen-activated protein kinase. J Biol Chem. 1996;271:23619-22.

5. Qian Y, Chai S, Liang Z, et al. KIF5B-RET fusion kinase promotes cell growth by multilevel activation of STAT3 in lung cancer. Mol Cancer. 2014;13:176.

6. Trupp M, Scott R, Whittemore SR, et al. Ret-dependent and -independent mechanisms of glial cell linederived neurotrophic factor signaling in neuronal cells. J Biol Chem. 1999;274:20885-94.

7. Kohno T, Ichikawa H, Totoki Y, et al. KIF5B-RET fusions in lung adenocarcinoma. Nat Med. 2012;18:375-7.

8. Lipson D, Capelletti M, Yelensky R, et al. Identification of new ALK and RET gene fusions from colorectal and lung cancer biopsies. Nat Med. 2012;18:382-4.

9. Ju YS, Lee WC, Shin JY, et al. A transforming KIF5B and RET gene fusion in lung adenocarcinoma revealed from whole-genome and transcriptome sequencing. Genome Res. 2012;22:436-45.

10. Chao BH, Briesewitz R, Villalona-Calero MA. RET fusion genes in non-small-cell lung cancer. J Clin Oncol. 2012;30(35):4439-41.

11. Ferrara R, Auger N, Auclin E, Besse B. Clinical and translational implications of RET rearrangements in non-small cell lung cancer. J Thorac Oncol. 2018;13(1):27-45.

12. Choudhury NJ, Drilon A. Decade in review: a new era for RET-rearranged lung cancers. Transl Lung Cancer Res. 2020;9(6):2571-80. doi:10.21037/tlcr-20-346.

13. Drilon A, Hu ZI, Lai GGY, et al. Targeting RET-driven cancers: lessons from evolving preclinical and clinical landscapes. Nat Rev Clin Oncol. 2018;15:150.

14. Gautschi O, Milia J, Filleron T, et al. Targeting RET in Patients With RET-Rearranged Lung Cancers: Results From the Global, Multicenter RET Registry. J Clin Oncol. 2017;35:1403-10.

15. Drilon A, Rekhtman N, Arcila M, et al. Cabozantinib in patients with advanced RET-rearranged nonsmall-cell lung cancer: an open-label, single-centre, phase 2, single-arm trial. Lancet Oncol. 2016;17:1653-60.

16. Yoh K, Seto T, Satouchi M, et al. Vandetanib in patients with previously treated RET-rearranged advanced non-small-cell lung cancer (LURET): an open-label, multicentre phase 2 trial. Lancet Respir Med. 2017;5:42-50.

17. Drilon A, Fu S, Patel MR, et al. A Phase I/lb Trial of the VEGFR-Sparing Multikinase RET Inhibitor RXDX-105. Cancer Discov. 2019;9:384-95.

18. Ribeiro M, Alessi JVM, Oliveira LJC, et al. Alectinib activity in chemotherapy-refractory metastatic RET-rearranged non-small cell lung carcinomas: A case series. Lung Cancer. 2020;139:9-12. 
19. Nakaoku T, Kohno T, Araki M, et al. A secondary RET mutation in the activation loop conferring resistance to vandetanib. Nat Commun. 2018;9:625.

20. Nelson-Taylor SK, Le AT, Yoo M, et al. Resistance to RET-Inhibition in RET-Rearranged NSCLC Is Mediated By Reactivation of RAS/MAPK Signaling. Mol Cancer Ther. 2017;16:1623-33.

21. Markham A. Selpercatinib: first approval[J]. Drugs. 2020;80(11):1119-24..Materials and methods.

22. Velcheti V, Bauer T, Subbiah V, et al. OA 12.07 LOXO-292, a Potent, Highly Selective RET Inhibitor, in MKI Resistant RET Fusion-Positive Lung Cancer Patients with and without Brain Metastases. J Thorac Oncol. 2017;12:1778.

23. Subbiah V, Gainor JF, Rahal R, et al. Precision Targeted Therapy with BLU-667 for RET-Driven Cancers. Cancer Discov. 2018;8:836-49.

24. Chen S, Zhou Y, Chen Y. Jia Gu; fastp: an ultra-fast all-in-one FASTQ preprocessor, Bioinformatics, Volume 34, Issue 17, 1 September 2018, Pages i884-i890, https://doi.org/10.1093/bioinformatics/bty560.

25. Li H, Durbin R. Fast and accurate long-read alignment with Burrows-Wheeler transform. Bioinformatics. 2010;26:589-95.

26. gencore. an efficient tool to generate consensus reads for error suppressing and duplicate removing of NGS data. Shifu Chen, Yanqing Zhou, Yaru Chen, Tanxiao Huang, Wenting Liao, Yun Xu, Zhihua Liu, Jia Gu bioRxiv 501502; doi: https://doi.org/10.1101/501502.

27. Li H, Handsaker B, Wysoker A, Fennell T, Ruan J, Homer N, Marth G, Abecasis G, Durbin R. 1000 Genome Project Data Processing Subgroup: The sequence alignment/map (SAM) format and SAMtools. Bioinformatics 25 2078-9, 2009.

28. Koboldt DC, Zhang Q, Larson DE, Shen D, McLellan MD, Lin L, Miller C, Mardis ER, Ding L, Wilson RK. VarScan 2: Somatic mutation and copy number alteration discovery in cancer by exome sequencing. Genome Res. 2012;22:568-76.

29. Talevich E, Shain AH, Botton T, Bastian BC. CNVkit: Genome-wide copy number detection and visualization from targeted sequencing. PLOS Computational Biology. 2014;12(4):e1004873.

30. Chen S, Liu M, Huang T, Liao W, Xu M. Jia Gu. GeneFuse: detection and visualization of target gene fusions from DNA sequencing data. International Journal of Biological Sciences, 2018; 14(8): 843-8. doi:10.7150/ijbs.24626.

31. 31.

32. Mayakonda A, Lin DC, Assenov Y, Plass C, Koeffler HP. 2018. Maftools: efficient and comprehensive analysis of somatic variants in cancer. Genome Resarch PMID: 30341162.

33. Golden Helix. GenomeBrowse ® visualization tool (Version 2.x) [Software]. Bozeman, MT: Golden Helix, Inc. Available from http://www.goldenhelix.com.

34. Zehir A, Benayed R, et al. Mutational landscape of metastatic cancer revealed from prospective clinical sequencing of 10,000 patients. Nat Med. 2017 Jun;23(6):703-13. doi:10.1038/nm.4333. 
35. Chalmers ZR, Connelly CF, Fabrizio D, et al. Analysis of 100,000 human cancer genomes reveals the landscape of tumor mutational burden. Genome Med. 2017;9:34. https://doi.org/10.1186/s13073017-0424-2.

36. Li W, Liu Y, Li W, et al. Intergenic breakpoints identified by DNA sequencing confound targetable kinase fusion detection in NSCLC[J]. Journal of Thoracic Oncology. 2020;15(7):1223-31.

\section{Figures}

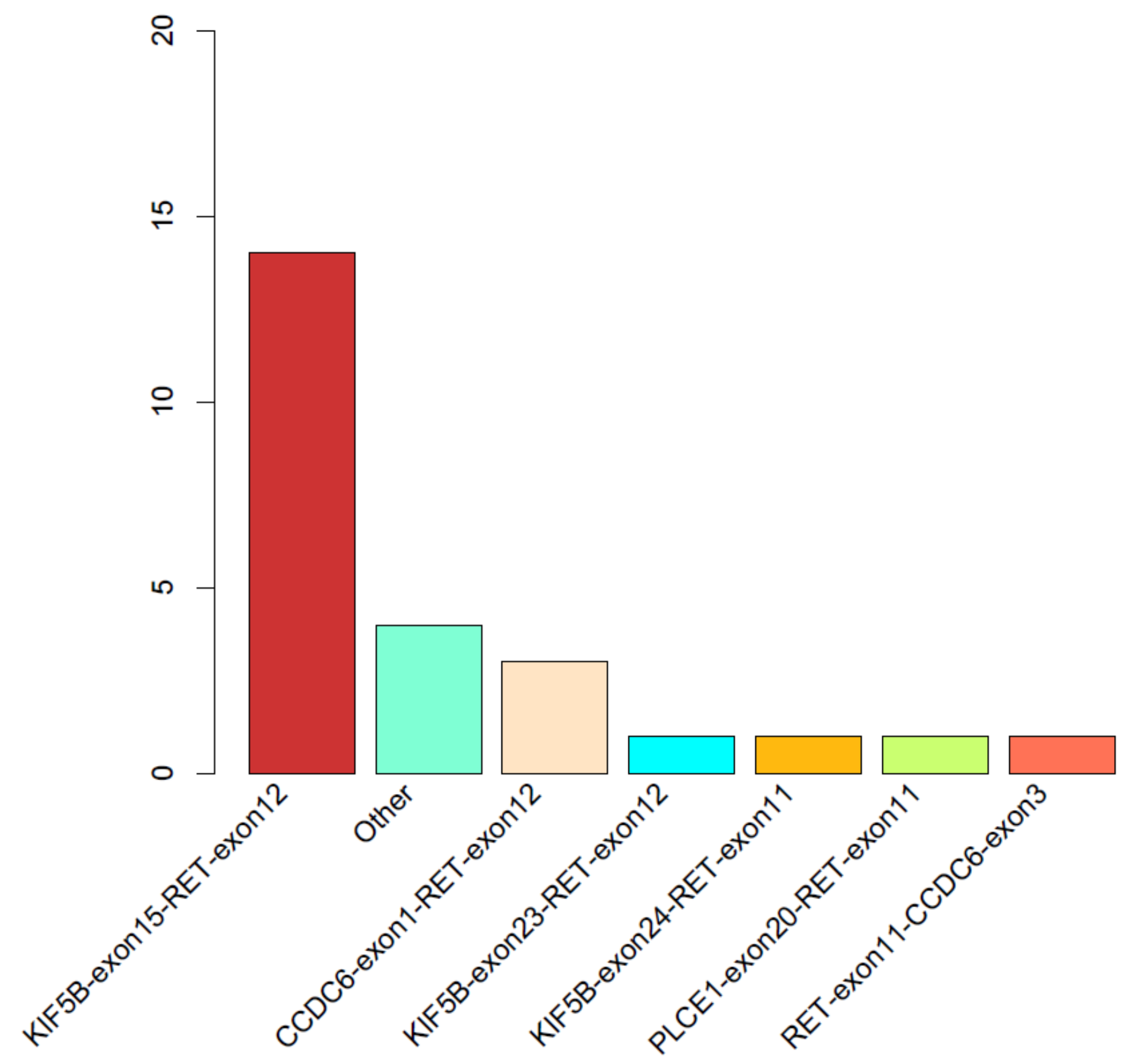

Figure 1 
The statistics of different RET rearrangement forms. The distribution of each RET fusion pattern identified in 25 NSCLC patients are shown in the barchart.

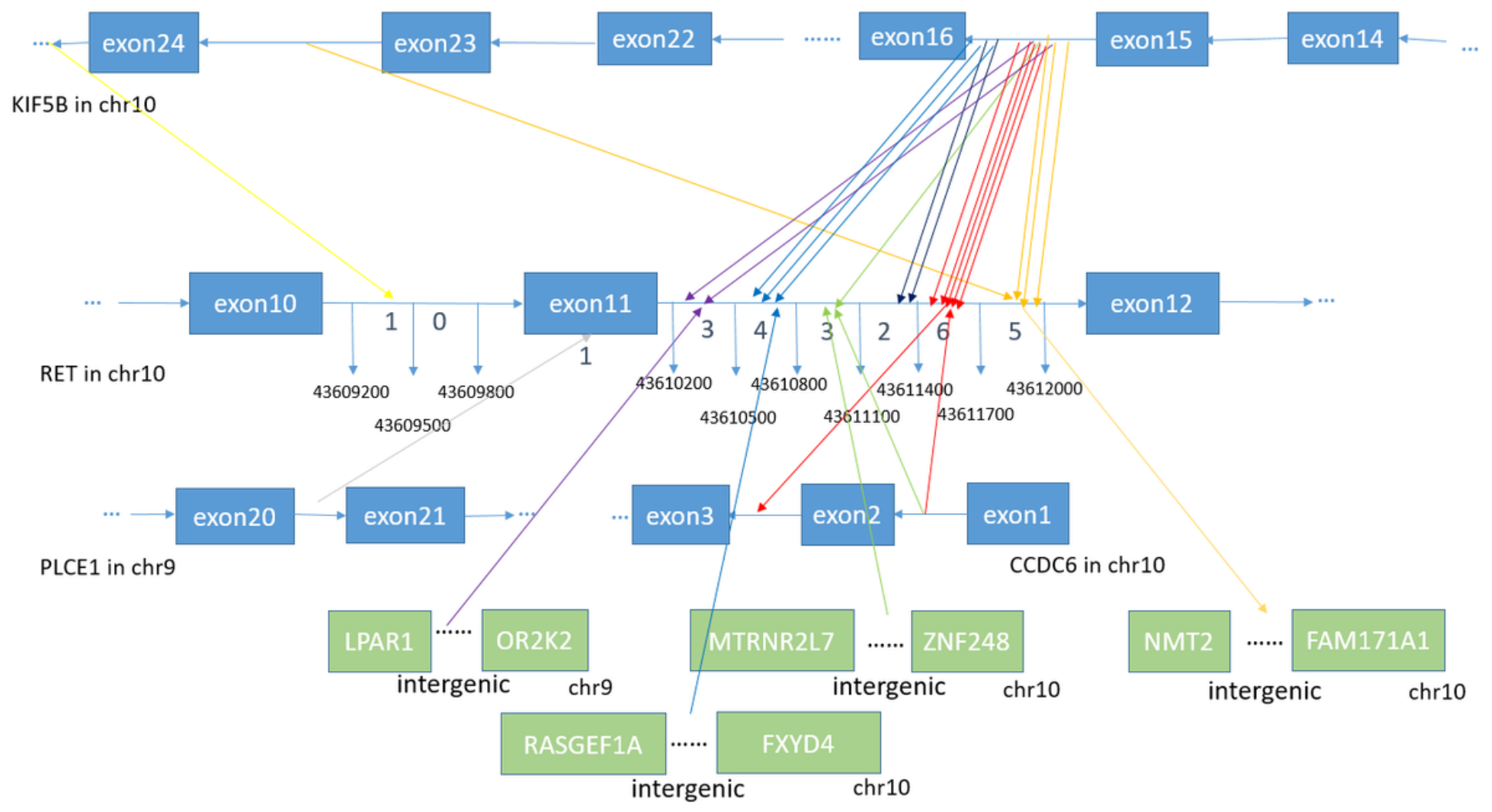

Figure 2

The breakpoint distribution in RET and the corresponding fusion partners. Each arrowed line represents one fusion event. The exact breakpoints of RET in GRch37 are shown in the middle panel, while the fused exons of RET fusion partners are shown in the top and bottom panels respectively. The sequences of KIF5B and CCDC6 are exhibited reversely (from right to left), while the sequences of RET and PLCE1 are represented in the forward direction. The genomic region of RET between 43609200 and 43609800 in intron 10 as well as region between 43610200 and 43612000 in intron 11 are divided into regions every $300 \mathrm{bp}$. Breakpoint positions in RET locate between 43609200 and 43609500 with an yellow arrow, between 43610200 and 43610500 with a purple arrow, between 43610500 and 43610800 with a blue arrow, between 43610800 and 43611100 with an green arrow, between 4361100 and 43611400 with a black arrow, between 4361400 and 43611700 with a red arrow, between 4361700 and 43612000 with a orange arrow, 


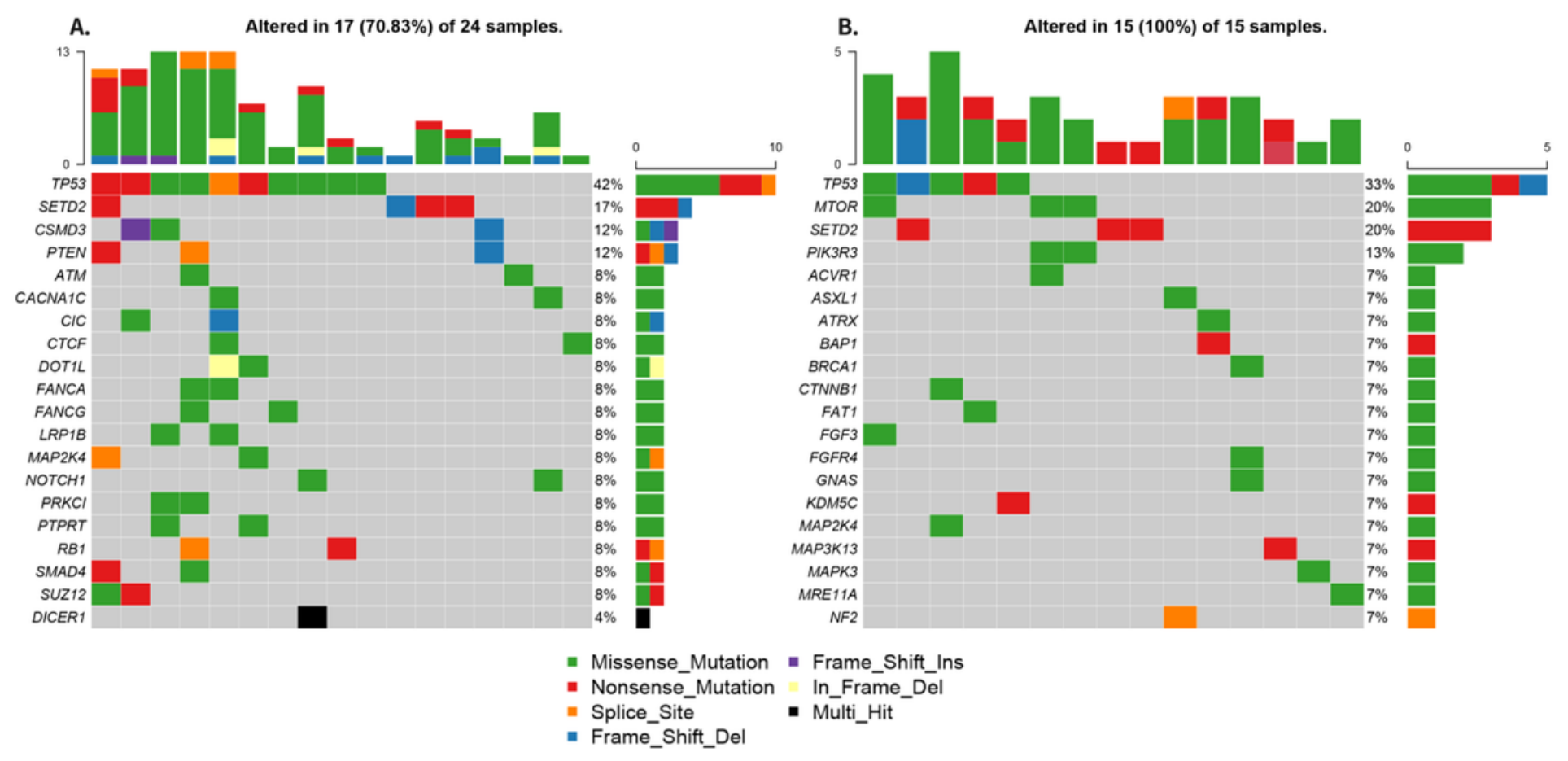

\section{Figure 3}

Mutational profiles of RET fusion-positive NSCLC patients. A. This is the oncoprint for the top 20 genes of the somatic SNVs and Indels of the 25 patients in our study. Somatic alterations included missense, nonsense, frameshift indel, in-frame indel, splice site, translation start site, and multi_Hit mutations. The genes are ranked by the frequency of the mutations across all samples. B. This is the oncoprint for the top 20 genes of the somatic SNVs and Indels of the 15 patients from the MSK-IMPACT study[34]. Somatic alterations included missense, nonsense, frameshift indel, in-frame indel, and splice-site mutations. The genes are ranked by the frequency of the mutations across all samples. 

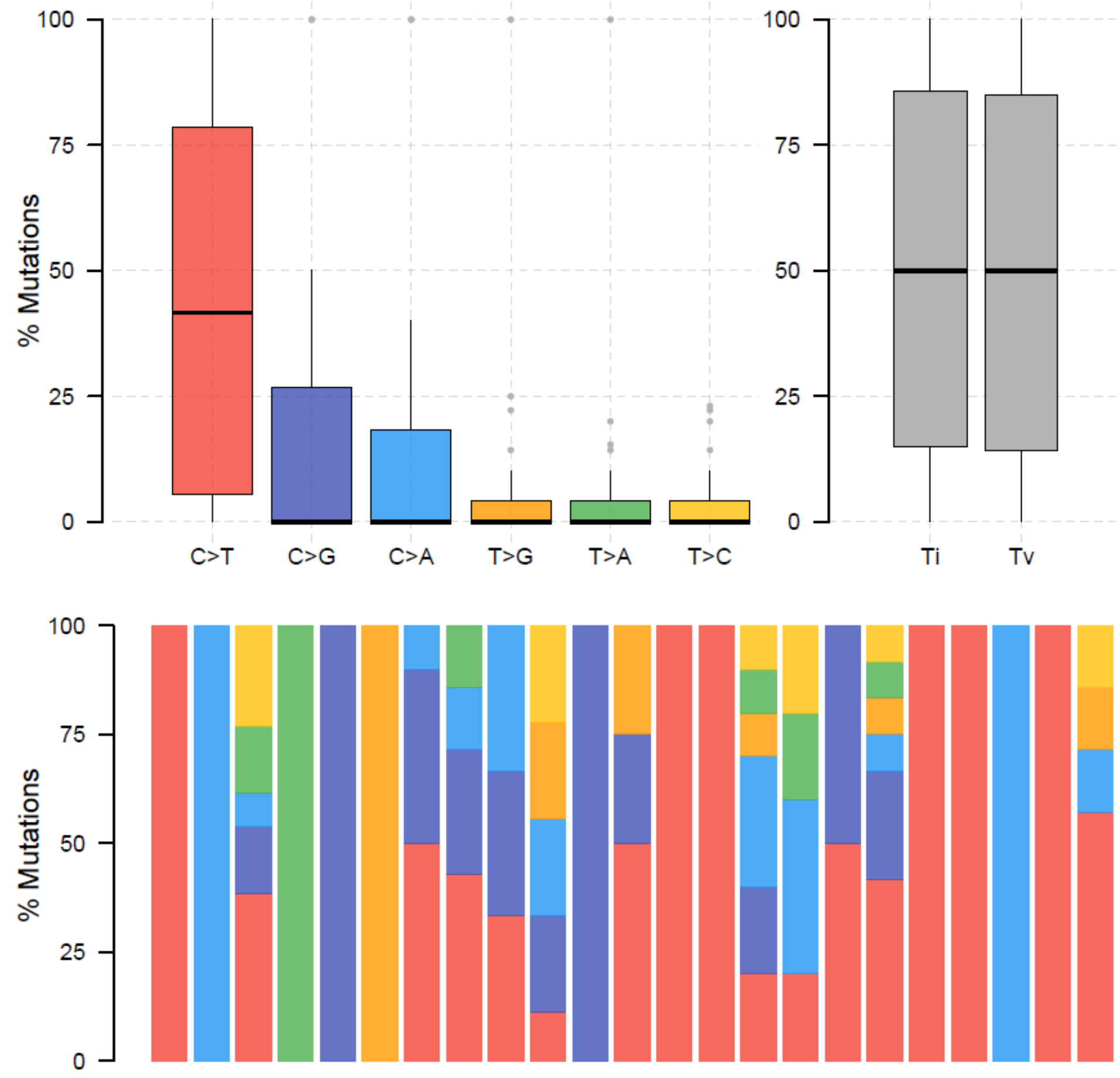

\section{Figure 4}

Mutational signatures of RET fusion positive NSCLC patients. SNPs are classified into transitions and transversions. Summarized data are visualized as a boxplot showing overall distribution of six different conversions (top) and as a stacked barplot showing the fraction of conversions in each sample (bottom). 
TP53 : [Somatic Mutation Rate: 41.67\%]

NM_000546

5

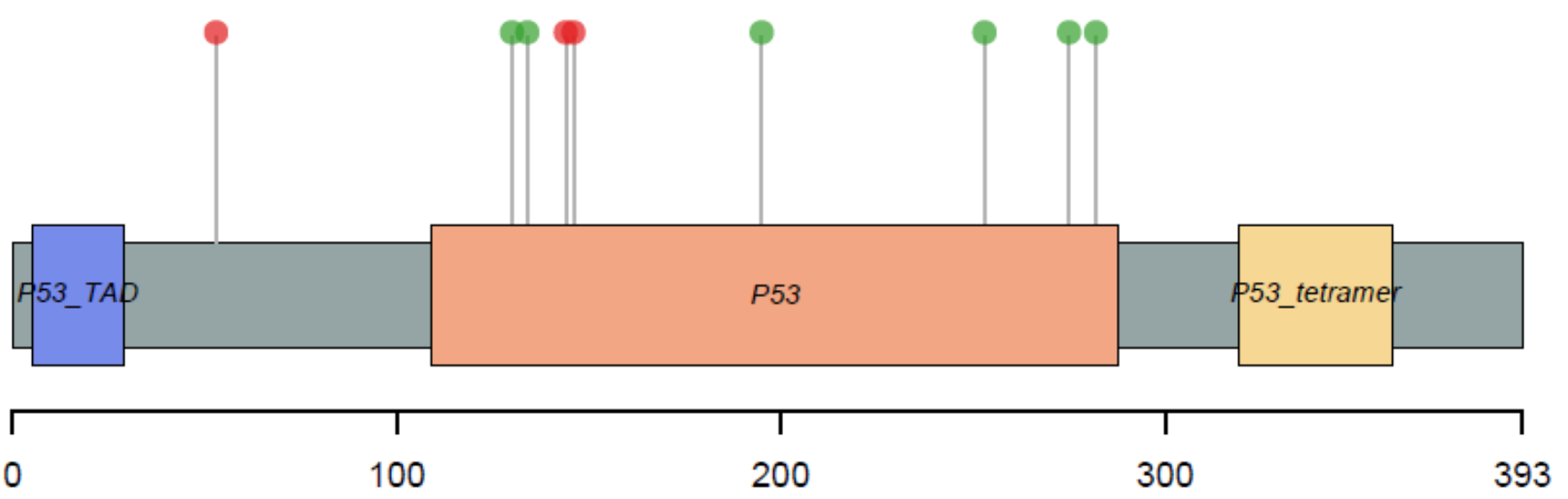

- Nonsense_Mutation

- Missense_Mutation

Figure 5

Protein variants resulted from TP53 mutations. Protein variants caused by TP53 mutations are displayed in the lollipop plot. These sites are considered to be mutational hot-spots. 


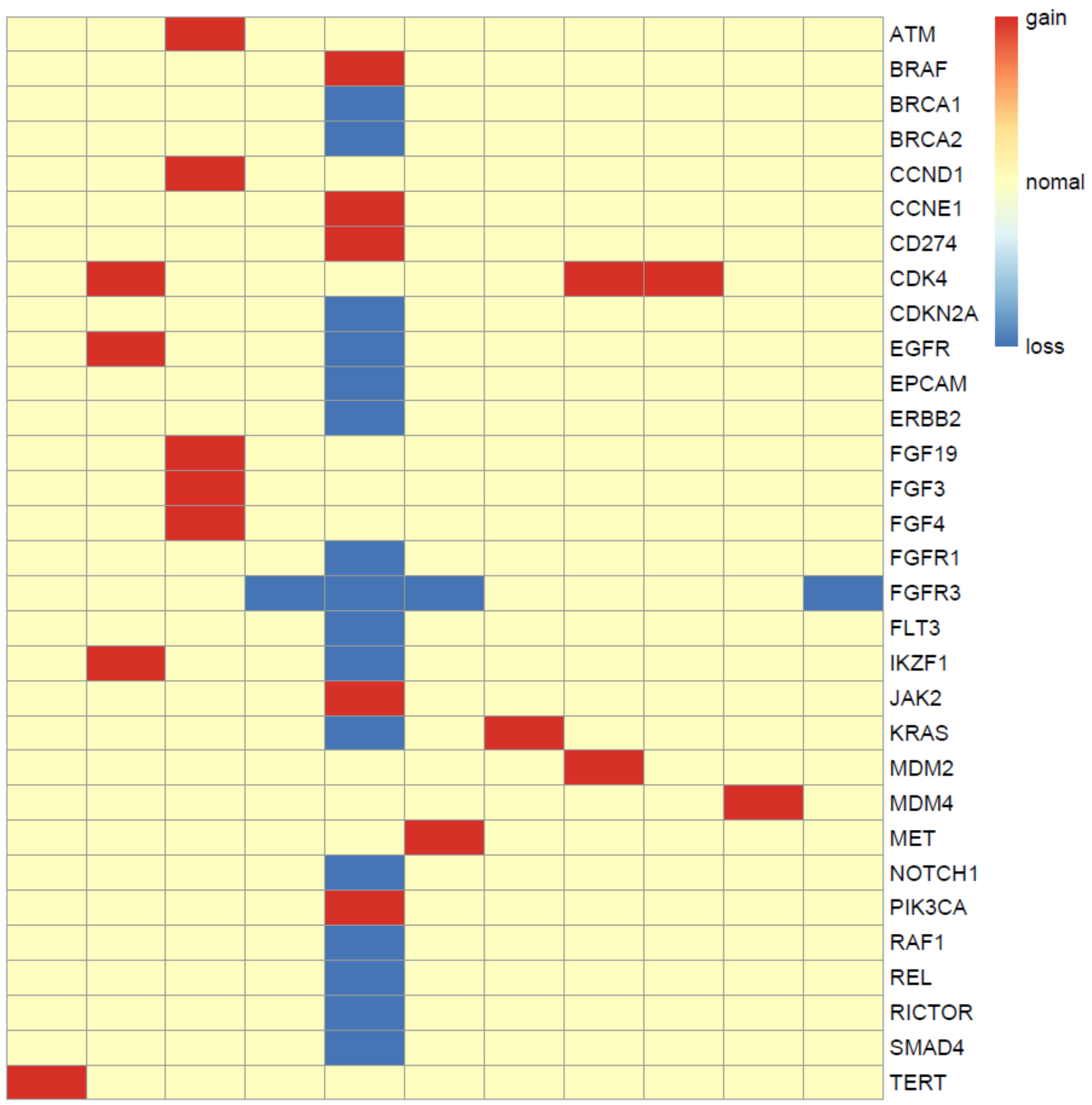

\section{Figure 6}

Copy number aberrations in 11 RET fusion-positive NSCLC patients. The names of the aberrant genes are shown in the $y$-axis, while $x$-axis represents individual patient. The type of copy number aberrations, including gain, normal and loss are indicated by red, yellow, and blue respectively. 